\title{
ESTRATEGIAS DE INTEGRACIÓN ACADÉMICA PARA ESTUDIANTES DE PRIMER INGRESO DE INGENIERÍA AGROFORESTAL EN URACCAN BILWI, 2009
}

Julia Pricela Salazar Francis ${ }^{[1]}$ Argentina García Solórzano ${ }^{[2]}$

\section{Resumen}

Esta investigación sobre Estrategias de integración académica para estudiantes de primer ingreso de Ingeniería Agroforestal en URACCAN Bilwi, 2009; es de tipo cualitativo, enfocado en el interaccionismo simbólico, lo que permitió explicar, describir y analizar diferentes estrategias que serán útiles y pertinentes para la integración académica de estudiantes de primer ingreso a la vida universitaria.

La principal discusión fue sobre cómo la universidad facilita la integración de estudiantes de nuevo ingreso, la metodología que utilizan los y las docentes en el proceso de enseñanza-aprendizaje relacionada con la integración de los estudiantes de nuevo ingreso, y lo que hacen los y las estudiantes de primer año de Ingeniería Agroforestal para integrarse a la vida académica en la universidad.

Se deben revisar reglamentos, el modelo pedagógico, las estrategias utilizadas como institución en la captación, la metodología y contenidos del curso propedéutico y la metodología de enseñanza-aprendizaje utilizadas en las aulas de clase y los planes estratégicos para poder contar con estrategias de pertinentes que faciliten integración estudiantil a la vida académica.

\section{Introducción}

En la URACCAN, después de catorce años de aportar de manera sustancial a la formación de profesionales autóctonos de calidad se han vivido diversas experiencias. A lo interno se han hecho estudios sobre rendimiento académico y sobre deserción estudiantil, lo que se ha visto de alguna manera como problemas asociados.

En este estudio sobre: Estrategias de integración académica para estudiantes de primer ingreso de Ingeniería Agroforestal en URACCAN Bilwi, 2009; se considera otro componente como es la integración a la vida académica, cómo se está haciendo y qué debería mejorarse.

[1] Docente de la Escuela de Liderazgo de la URACCAN Recinto Bilwi.

[2] Secretaria Académica de la URACCAN Recinto Bilwi. Máster, tutora. argentina_garcia@yahoo.com 
Algunos resultados obtenidos como producto del trabajo son:

La estrategia más utilizada por los docentes en las aulas es la exposición frente a grupo sin coloquio, en la que la mayor participación la desempeña el docente, relegando a un segundo plano la intervención de los estudiantes.

Entre las estrategias y mecanismos que emplea la universidad para facilitar la integración académica de los estudiantes de nuevo ingreso se destaca la implementación de los cursos propedéuticos en la que se aplica la orientación vocacional que es una manera de preparar psicológicamente al estudiante para que se integre con éxito a la vida académica en la universidad.

Los estudiantes, mujeres y hombres, de la carrera de Ingeniería Agroforestal para integrarse a la vida universitaria cumplen con sus tareas, buscan información en la biblioteca, trabajan en equipo, participan en jornadas científicas, realizan clases prácticas y participan en actividades deportivas.

Con los resultados de este estudio se proporciona aportes para el mejoramiento de estrategias institucionales, pedagógicas y estudiantiles que faciliten una integración académica exitosa del estudiantado en la vida universitaria.

\section{Objetivos}

\section{Objetivo general}

Valorar las estrategias que se utilizan en la carrera de ingeniería Agroforestal de URACCAN Bilwi para facilitar la integración académica de los estudiantes a la vida universitaria y su relación con el rendimiento académico durante el año 2009.

\section{Objetivos específicos:}

1. Caracterizar a estudiantes de primer año de Ingeniería Agroforestal.

2. Describir las estrategias que utilizan los y las docentes para la integración académica de estudiantes de primer ingreso en la carrera de Ingeniería Agroforestal de URACCAN Bilwi.

3. Identificar estrategias de integración a la vida universitaria por parte de los estudiantes y de la institución del primer ingreso de la carrera de Ingeniería Agroforestal de URACCAN Bilwi.

4. Determinar la relación entre el proceso de integración a la vida académica y el rendimiento académico de estudiantes del primer ingreso en la carrera de Ingeniería Agroforestal en la URACCAN Bilwi. 


\section{CIENCIAS SOCIALES}

5. Proponer algunas estrategias que contribuyan al mejoramiento de la integración de estudiantes de primer ingreso de la carrera de Ingeniería Agroforestal

\section{Metodología}

Integración del estudiante al sistema universitario:

Díaz Peralta (2008:5) plantea que: "La formación de expectativas sobre las condiciones de la vida estudiantil o académica puede conducir a decepciones tempranas en el caso que lo esperado sea mayor que lo recibido". Ello pone en movimiento una serie de interacciones que llevan a la deserción.

Al respecto, Sanz R. (2005:11), manifiesta que "los profesores pueden ayudar a los estudiantes a reconocer que su aprendizaje en la clase está relacionado con sus vidas fuera de las clases". Por eso es importante dedicar un tiempo a dialogar con los estudiantes sobre lo que aprenden y cómo ayudarles a hacer conexiones, integrar y aplicar lo que están aprendiendo a su vida o mundo real.

Así, Díaz C. (2008:6), explica que no es: "Si nosotros respondemos, ellos acudirán a la tutoría"; y que más bien es "si nosotros les preguntamos y respondemos intencionalmente a lo que necesitan, ellos acudirán a la tutoría".

Chickering y Reisser (1993:236) agregan que el hecho de adoptar un sistema de valores, no tiene nada que ver con un sistema rígido de creencias, sino más bien: "un movimiento hacia el fomento de la responsabilidad personal y de los demás y la habilidad para regirse por principios éticos".

En otras tareas tutoriales Coriat \& Sanz (2005:56) argumentan que en ocasiones, el profesorado universitario se queja o se lamenta porque no comprende a sus estudiantes. Algunos creen que los estudiantes de hoy no consideran que la asistencia a clase sea importante, no hacen las tareas asignadas o no prestan atención en clase.

Con estudiantes diversos y necesidades diversas, los programas de formación tienen que ayudar a los tutores a desarrollar las actitudes y habilidades necesarias para enfrentarse con garantías de éxito en sus estudios.

Agregan los mismos autores que se está hablando de un rediseño de la actividad docente. Pasar de una idea pasiva centrada sólo en el proceso de enseñanza a una idea activa centrada en lo que el alumno aprende, es decir, lo que va a ser capaz de conocer, lo que va a ser capaz de hacer y las actitudes que va a adoptar para continuar aprendiendo de forma autónoma a lo largo de toda su vida. 
Al respecto, Díaz C. (2008:12) cita esta frase de Frankola (2001), quien afirma:

la falta de supervisión, motivación, problemas con la tecnología, falta de apoyo a los estudiantes, las preferencias de aprendizaje, diseño inadecuado de los cursos e instructores inexpertos, son las principales causas del fracaso estudiantil, en la educación a distancia.

Bolívar (2003:7) argumenta que las competencias como trabajo en equipo, desarrollo de la capacidad de síntesis, crítica, pensamiento comparativo, analítico, comunicación y expresión oral y escrita, por citar algunas, son claves para el desarrollo personal, académico y profesional de los estudiantes el profesor adquiere el papel de acompañante, de supervisor y guía del aprendizaje, para alcanzar objetivos y competencias previamente definidas.

Para Casado R. \& Ruiz M. (2008:1), una forma de facilitar a sus estudiantes el paso a la Educación Superior, es "que ante la desorientación en la que se encuentran los y las estudiantes de nuevo ingreso y la detección de necesidades tanto educativas como formativas, hemos creído conveniente la puesta en marcha del Programa Mentor: tutorías entre compañeros/as".

Plantean que la Universidad de Burgos en España quiere introducir una herramienta que permita su adaptación en los diferentes ámbitos: académico, social, personal y profesional. Agregan: "Se trata de una actividad que promueve la participación activa de los estudiantes en la vida universitaria y además completa su formación para la adaptación a diferentes contextos".

Al respecto, Tinto V. (1975:9) plantea "Los estudiantes actúan de acuerdo con la teoría de intercambio en la construcción de su integración social y académica", es decir, si el estudiante percibe que los beneficios de permanecer en la universidad son mayores que los costos personales, entonces el estudiante permanecerá en la institución.

En el sentido contrario, si observa que otras actividades son percibidas como fuentes de recompensas más altas, el estudiante tenderá a desertar. El mismo autor sugiere que una buena integración es uno de los aspectos más importantes para la permanencia, y que esta integración depende de las experiencias durante la permanencia en la universidad, las experiencias previas al ingreso universitario y las características individuales que, por otro lado, son susceptibles a las políticas y prácticas universitarias.

Ruiz Estrada H. \& Mayet Moreno R. L. (2005:10) valoran esa idea y expresan en el Modelo de la Integración del Estudiante de Tinto V. que hay dos procesos que determinan el éxito escolar: la integración académica y la integración social. 
¿Qué favorece la integración académica?

Entre los factores académicos que dependiendo de cómo sean atendidos, Díaz P. (2005) pueden convertirse en una ventaja para la integración académica, según él estas son: "la orientación profesional, el desarrollo intelectual, el rendimiento académico, los métodos de estudios, los procesos de admisión, los grados de satisfacción de la carrera y la carga académica".

Ruiz Estrada H. \& Mayet-Moreno R. L. (2005) afirman que el fracaso escolar a nivel de licenciatura es un síndrome bien conocido a nivel nacional; aún cuando el estudiante que ingresa a la universidad es un "sobreviviente" de varios procesos de selección, su adaptación a los estudios universitarios no está asegurado con el correspondiente abandono de la carrera.

Miguel et al. (2004), sobre la integración académica, plantean que "es necesario ofrecer a los estudiantes/as de nuevo ingreso la orientación y asesoramiento necesarios para facilitar su integración académica y social en la institución y, en la medida de lo posible, lograr el éxito en sus estudios"

Esto implica según los autores, facilitar el desarrollo de habilidades y proporcionar estímulos para el fomento de la reflexión, el diálogo en el ámbito académico, la autonomía, la crítica, promover el desarrollo de actitudes y valores de compromiso, responsabilidad, respeto y solidaridad, ofrecer claves para su futuro y desempeño profesional, desarrollar competencias técnicas (saber), sociales, de participación (saber ser), metodológicas (saber hacer), de dirección y trabajo en grupo y orientar el aprendizaje, anticipándose a las dificultades.

El propósito de lo anterior es facilitar la transición del bachillerato a la universidad. Así mismo tiene el propósito de fomentar la participación en la vida universitaria y en el uso de sus servicios, proporcionar refuerzos académicos y ayudarles a superar las exigencias académicas, orientar para la formación permanente, y disminuir el abandono universitario y el fracaso escolar y aumentar el número de estudiantes de nuevo ingreso.

\section{Integración social y compromiso institucional}

Cuando la integración social del alumno con su grupo es débil, Tinto V. (1975), afirma que el estudiante no alcanza a acomodarse y tiende a desertar, a no ser que encuentre por lo menos algún compañero con el que se comprenda y así pueda terminar el curso, apoyado en esta relación, pero la situación en estas condiciones inclina la balanza poco a poco hacia la deserción. 
Díaz C. (2008) aludiendo a otros autores, expresa que Anderson (1981), Braxton y Brier (1989), Pascarella y Chapman (1983a, 1983b), Pascarella et al. (1986), Pascarella y Terenzini (1985) reportaron que la integración académica es más fuerte que los compromisos o metas institucionales; por tanto, los compromisos institucionales tienen un efecto indirecto sobre la permanencia a través de la integración social.

En este nuevo paradigma es más importante aprender a aprender, aprender a transformar información a nuevos conocimientos, y aprender a transferir nuevos conocimientos a las aplicaciones.

\section{Compromiso académico e identificación profesional}

Bayer \& Shah (1967), argumentan que el bajo rendimiento puede provenir de dos fuentes: capacidad intelectual insuficiente o débil compromiso académico. Si se trata de lo primero, la deserción es forzosa y poco remedio hay para ella. En varios estudios la habilidad intelectual se ha erigido como la variable predictora más importante en el fenómeno desertivo.

\section{Estrategias de enseñanza que favorecen la integración académica}

De acuerdo al documento Actividades para estudiantes de nuevo ingreso de la Universidad Politécnica de Madrid (2009), se organizan diversas actividades de acogida para los estudiantes de nuevo ingreso, que pretenden facilitar el salto de los estudios de secundaria a la Universidad, tanto los referidos a los conocimientos como los relativos a la administración y gestión académica.

Guadamuz (1990), afirma que existe en la enseñanza lo que se conoce como estrategia didáctica que para, este autor la estrategia didáctica "es una disciplina, un campo del conocimiento educativo que se ocupa de los procesos de enseñanza -aprendizaje en contexto institucional para alcanzar la formación".

Díaz \& Frida (2001), expresan que:

Las estrategias para orientar la atención de los estudiantes son aquellos recursos que el profesor o el diseñador utiliza para focalizar y mantener la atención de los aprendices durante una sesión, discurso o texto. Los procesos de atención selectiva son actividades fundamentales para el desarrollo de cualquier acto de aprendizaje.

Dubón M. \& Zambrana R. (2006), citando a Nisbeth J. (1998:2), las estrategias de enseñanza están referidas a:

Las formas para trabajar en función de realizar las diferentes actividades de aprendizaje. Por ejemplo: el estudio de casos, la resolución de problemas, el incidente crítico, 
el debate, la exposición con o sin coloquio, simulaciones, el análisis de materiales, la acción autorizada.

\section{Resultados y discusión}

Caracterización de estudiantes de primer año de Ingeniería Agroforestal

El 8o por ciento de los estudiantes (mujeres y hombres) de la carrera de Ingeniería Agroforestal son de la etnia miskita. Proceden de las comunidades ubicadas en los municipios de Waspam, Prinzapolka y Puerto Cabezas, en su totalidad son de escasos recursos económicos y han cursado sus estudios de Educación Secundaria en la escuelas públicas de la región y algunos en las secundarias rurales, donde la enseñanza no es de buena calidad.

Es por esto que algunos estudiantes realizan trabajos menores para poder sufragar los gastos de su estudio en la universidad, otros son apoyados por sus padres que en la medida de sus posibilidades les brindan algún tipo de ayuda económica y material. Una mínima parte tienen trabajo de medio tiempo.

\section{Descripción de las estrategias implementadas en el aula de clase}

En el desarrollo de las clases se utilizan las siguientes:

\section{La exposición frente a los grupos}

Los docentes preparan a los estudiantes para realizar la exposición de un determinado tema utilizando un datashow. El o la docente procede a realizar algunas preguntas sobre el tema y generalmente es el mismo estudiante quien responde entonces la clase la realiza con seis u ocho estudiantes descuidando a los demás que no están motivados a participar en la clase.

Durante este tiempo algunos estudiantes se dedican a escuchar y algunos toman nota o apuntes, otros están distraídos realizando otras actividades (escuchando música, jugando con el teléfono celular, conversando con otros compañeros, entre otras). Como resultado de esta estrategia es que no se logró el desarrollo de las habilidades y conocimientos en los estudiantes.

\section{Tratamiento docente a estudiantes que no tienen dominio del español}

Tinto (1975) plantea que el estudiante si bien puede lograr una integración y cohesión social dentro de la institución, no alcanza un rendimiento aceptable, por lo cual se ve obligado a retirarse. Pareciera que no hay relación entonces entre la integración y el rendimiento académico. Se ve sencillo el razonamiento: a mejor integración mejor 
rendimiento académico, pero no siempre es así. La integración se complementa con las estrategias didácticas acertadas que utilizan los docentes.

¿Qué han hecho los estudiantes para integrarse a la vida universitaria?

Por su parte, los estudiantes expresaron que ellos luchan como personas para mejorar su nivel académico, adaptarse a la vida universitaria, dedicar más tiempo y ser mejores profesionales con calidad para servir a su patria.

Díaz C. (2005), cita a Frankola (2001), quien reafirma que la falta de supervisión, motivación, los problemas con la tecnología, la falta de apoyo a los estudiantes, las preferencias de aprendizaje, el diseño inadecuado de los cursos e instructores inexpertos, son las principales causas que explica la deserción. En este sentido Tinto (1975:8) explica el proceso de permanencia en la Educación Superior "como una función del grado de ajuste entre el estudiante y la institución, adquirido a partir de las experiencias académicas y sociales (integración)". Asocia esta concepción a la base de la teoría de intercambio que se encuentra en el principio de que los seres humanos evitan las conductas que implican un costo de algún tipo para ellos y buscan recompensas en las relaciones, interacciones y estados emocionales.

\section{Estrategia en la universidad}

Entre las estrategias y mecanismos que emplea la universidad para facilitar la integración académica de los estudiantes de nuevo ingreso se destaca la implementación de los cursos propedéuticos en la que se aplica la orientación vocacional.

De esta manera preparar psicológicamente al estudiante para que se integre con éxito a la vida universitaria, además, se le brinda atención a través del área de bienestar estudiantil en donde se atiende a estudiantes que presentan mayores problemas y dificultades académicas y de adaptación.

Se ofertan clases de tutoría, capacitación pedagógica a docentes, charlas a estudiantes, así también, se integran a los estudiantes en algunas actividades de los institutos y en la coordinación. Se les brinda orientaciones y oportunidades para participar en actividades recreativas y deportivas a nivel de recinto. Existe la política de becas, ya sea de transporte, régimen de internado entre otros.

Al respecto, Rafael Sanz Oro (2005) manifiesta: "los profesores pueden ayudar a los estudiantes a reconocer que su aprendizaje en la clase está relacionado con sus vidas fuera de las clases". Por eso es importante dedicar un tiempo a dialogar con los estudiantes sobre lo que aprenden y cómo lo así como ayudarles a hacer conexiones, integrar y aplicar lo que están aprendiendo a su vida o mundo real. 


\section{Conclusiones}

En la universidad existe como formas de integración académica a la vida universitaria: los cursos propedéuticos, el sistema de becas y acciones afirmativas para jóvenes sobre todo indígenas de las zonas más marginadas.

Las estrategias utilizadas por los docentes en la carrera de Ingeniería Agroforestal de la URACCAN carecen de pertinencia para la integración académica de los estudiantes de primer ingreso.

La mayor parte de las estrategias utilizadas por los docentes son de corte tradicional y no están centradas en el estudiante.

La integración a la vida académica universitaria, los estudiantes la hacen de manera inconsciente, no intencionada por lo que requieren de orientación, revisión y de ampliación de la gama de actividades.

Existe una relación directa entre el proceso de integración a la vida académica y el rendimiento académico de estudiantes del primer ingreso en la carrera de Ingeniería Agroforestal de la URACCAN Recinto Bilwi, lo que también se asocia a la deserción sobre todo en el primer semestre.

La comunicación es fundamental en la orientación a los y las estudiantes lo que les facilitaría la integración.

Los documentos oficiales de la universidad tales como el Modelo de Universidad Comunitaria, el modelo pedagógico y las diferentes normativas no consideran la etapa de adaptación para la integración de los y las estudiantes a la vida académica.

\section{Recomendaciones}

- En términos generales se recomienda a la URACCAN la revisión de sus documentos oficiales para integrar la etapa de adaptación de estudiantes.

- Realizar revisión del currículo de la carrera de Ingeniería Agroforestal para determinar la pertinencia de las estrategias metodológicas recomendadas y actualizarlas considerando la integración académica de estudiantes de nuevo ingreso.

- Capacitar a los docentes sobre el uso de estrategias metodológicas de integración que permita un mayor índice de retención y aprobación en los estudiantes de nuevo ingreso a la carrera de Ingeniería Agroforestal. 
- En los procesos de acompañamiento en la carrera identificar y fortalecer el proceso de integración de los estudiantes a la vida académica de la universidad.

- La comunidad universitaria en su conjunto, apoyar y participar en las acciones de integración a la vida universitaria para los nuevos ingresos

- Cada docente de la carrera debería tomarse siempre un espacio de tiempo para el diálogo y la atención individual.

- Aplicar estrategias metodológicas pertinentes que promueven la integración activa y participativa del estudiantado de la carrera de Ingeniería Agroforestal.

- Fomentar en los estudiantes de primer ingreso de la carrera de Ingeniería Agroforestal hábitos de estudio a través de la asignación de trabajos e investigaciones de manera controlada y sistemática.

- Hacer uso de diversos recursos didácticos del entorno y tecnológicos que permita al estudiante entrar en contacto con el nuevo concepto y conocimiento en estudio.

- Promover en los estudiantes la participación en las actividades deportivas, culturales y recreativas.

- Establecer horarios de estudio y cumplirlas de manera disciplinada con el fin de mejorar su rendimiento y aprovechamiento académico en la carrera de Ingeniería Agroforestal.

- Participar en las actividades deportivas, sociales y culturales que impulsa la universidad para fomentar el espíritu de sociabilidad y de integración a la vida de la universidad.

- Para la integración es importante que la universidad prepare al docente para enseñar a los estudiantes que no son monolingües y que usan más su lengua materna.

- El Gobierno estudiantil debería promover acciones que conlleven a facilitar la integración académica de manera activa y participativa a través de promover los grupos de estudios con la asignación de estudiantes monitores, esto con la coordinación de los docentes de las diferentes áreas de estudio.

- El Gobierno estudiantil que se deberá organizar actividades deportivas, recreativas y culturales en las que se involucren con más beligerancia a los estudiantes de nuevo ingreso. 


\section{CIENCIAS SOCIALES}

\section{Lista de referencias}

Aguado Odina María Teresa. (1991). La educación Intercultural: conceptos, paradigmas y realizaciones. Lecturas de pedagogía diferencial. Madrid.

Aguilar Rivera María del Carmen. (2004). La transición a la Vida Universitaria: Éxito, Fracaso, Cambio y Abandono. Argentina

Aguirre, R. y Zambrana R. (2009). Ponencia en el VIII Seminario Taller Interanual Retos y Desafíos en la formación de Docentes. Managua - UNAN.

Bolívar, A. (2003). Diseño de planes de estudio de las titulaciones, Vicerrectorado de Planificación, Calidad y Evaluación Docente. Universidad de Granada.

Cabrera Castillo Henry Giovanni. (2008). Estudios realizados: Noveno semestre de Licenciatura en Bío química (Universidad del valle, sede Cali - Colombia.

Casado Muñoz, Raquel; Ruiz Franco, Mónica. (2008). Programa Mentor: Tutorías Entre Compañeros/as. Universidad de Girona. España

Coriat, M. \& Sanz, R. (Eds.). (2005 pp56). Orientación y Tutoría en la Universidad de Granada, Granada, Editorial, Universidad de Granada.

Chickering, A.W. \& Reisser, L. (1993). Education and identity, San Francisco: Jossey-Bass.

Delors, J. (1996). La educación encierra un tesoro. Editorial Santillana. Ediciones UNESCO. México, D.F.

Díaz-Barriga, A. Frida, Hernández, R. Gerardo. (2001). Estrategias docentes para un aprendizaje significativo: una interpretación constructivista. Capítulos 5 y 6.Editorial Mc Graw Hill. México. Segunda edición: octubre. 465 páginas

Díaz, F. Hernández, G. (1999). Estrategias docentes para un aprendizaje significativo una interpretación constructivista, McGRAW-HILL, México.

Díaz Peralta Christian. (2008). Estudios Pedagógicos XXXIV, N²: 65-86.

Dubón, M. \& Zambrana R. (2006). Didáctica Contemporánea de la Educación Superior I Managua-UNAN.

Gasteiz Victoria. (2005). Tratamiento Lingüístico. Proyecto Educativo.

López Cartagena Maribel. (2008). La educación intercultural: sinónimo de tolerancia y unidad. Directora de Título V. Universidad Interamericana de Puerto Rico. Recinto de Barranquitas.

Marqués, P. Didáctica. (2001). Los Procesos de Enseñanza y Aprendizaje. La Motivación. Departamento de pedagogía aplicada, Facultad de educación, UAB. 
Ruiz Estrada H. y, Mayet-Moreno R. L. (2005). El éxito académico de estudiantes de física en relación con su nivel de razonamiento y actitud.

Ruvalcaba, H. (2004). Aprender a Aprender.- Estrategias de enseñanza aprendizaje: Extraído el 20 de noviembre 2008 de http//www.uag.mx/68.

Salmi, Jamil. (2001). La Educación Superior en un punto decisivo. Ponencia presentada al Congreso de "Educación Superior, desafío global y respuesta nacional. Universidad de Los Andes, Santa Fe de Bogotá.

Tedesco Juan C. (2000). El nuevo pacto educativo calidad para todo.

Tinto, Vincent. (1975). La deserción en la Educación Superior: Síntesis de las bases teóricas de las investigaciones recientes, en: Review of Educational Research, Vol. 45, No. 1, USA, , pp. 89-195 (trad. de Carlos María de Allende)

Universidad Politécnica de Madrid. (2009). Actividades para estudiantes de nuevo ingreso. 